\title{
High-throughput spectroscopic viscosity measurement of nanocomposite sols with ETC-effect
}

\author{
Gerd Schäfer · Helmut K. Schmidt
}

\begin{abstract}
A high-throughput method for viscosity measurement was developed and tested for nanocomposite sols with an easy-to-clean (ETC) effect. The method is based on doping of sols with viscosity sensitive fluorescent dye 4,4'-bis(2-benzoxazolyl)-stilbene (BOS) and acquisition of fluorescence intensity data. The spectroscopic data were correlated with viscosity data derived from mechanical measurements with a rotational viscometer and show an exponential correlation of both mechanical and spectroscopic measurement methods in the relevant data space of 3-5 mPa.s. For application of the spectroscopic viscosity measurement as a high-throughput assay doping of sols with BOS slurry was carried out with an automated liquid handling system, and spectroscopic measurements were performed with a fluorescence microplate reader.
\end{abstract}

Keywords High-throughput viscosity measurement . Spectroscopic viscosity measurement - Viscosity sensitive fluorescent dye $\cdot$ Nanocomposite sol

\section{Introduction}

The viscosity is a very important parameter for controlling a coating process. In particle containing coating systems, the particle to particle interaction can influence the rheological parameters strongly, especially, if the particles are nano scale. Conventional viscosity measurements are performed for example with cone and plate type or rotational viscometer [1]. Such methods are well established but have several

G. Schäfer · H. K. Schmidt ( $\square)$

Institut für Neue Materialien gem. GmbH,

Saarbrücken, Germany

e-mail: h.schmidt@nm.uni-saarland.de disadvantages in the light of fast and efficient screening of nanocomposite sol viscosity, e.g. the volume necessary for conventional methods is in the range $2-20 \mathrm{ml}$, measurements are sequential, and the equipment has to be cleaned carefully after each measurement.

High-throughput methods for viscosity measurements described in literature are a "tuned-fork" viscometer developed according to patent [2] and a viscosity sensitive fluorescent probe applied for in situ investigation of UV-curing processes [3]. The usability of viscosity sensitive dyes, mainly derivatives of julolidine, was demonstrated also in the field of life sciences where spectroscopic viscosity measurement methods for blood plasma [4] and cell membranes [5] were established. Further studies used viscosity sensitive fluorescent probes as a versatile tool for the study of curing kinetics of epoxy resins [6].

However, the use of a high-throughput method for the optimization of material properties of sol-gel derived hybrid nanocomposites has not yet been described.

In this work we present the application of a spectroscopic high-throughput assay for viscosity measurement of an organically modified nanocomposite sol mixed with different amounts of 2-propanol. The chosen nanocomposite sol is a versatile material for anti-adhesive coatings on several substrate materials [7].

\section{Experimental section}

The nanocomposite sol was prepared from a mixture of $31.3 \mathrm{wt} . \%$ methyltriethoxysilane (MTEOS), $36.6 \mathrm{wt} . \%$ tetra ethyl ortho silicate (TEOS), 9.2 wt.\% aqueous silica sol (Bayer Levasil ${ }^{\circledR}$ 300/30\%), and 22.9 wt.\% (heptadecafluoro -1,1,2,2-tetrahydrodecyl)-triethoxysilane (FTS). MTEOS, TEOS, and FTS were hydrolized with $40 \mathrm{wt} . \%$ formic acid 
Table 1 2-propanol content and viscosity data derived from measurements with a rotational viscometer of nanocomposite sol samples used in this study

\begin{tabular}{lll}
\hline Sample & 2-propanol content [wt.-\%] & $\eta[\mathrm{mPa} \cdot \mathrm{s}]$ \\
\hline 1 & 0 & 4.7 \\
2 & 20 & 4.0 \\
3 & 25 & 3.8 \\
4 & 33.3 & 3.3 \\
5 & 50 & 3.0 \\
\hline
\end{tabular}

$\left(R_{\mathrm{OR}}=2\right)$, filtrated with a $5 \mu \mathrm{m}$ filter and divided into five portions (see Table 1).

The conventional viscosity measurements were carried out with a Physica Rheolab MC 20 rotational viscometer (measuring head: $\mathrm{Z1}$, sample volume: $17 \mathrm{ml}$ sol, sheer rate: $D=0-600 \mathrm{~s}^{-1}$ in $120 \mathrm{~s}$ and $600-0 \mathrm{~s}^{-1}$ in $120 \mathrm{~s}$, $T=20 \pm 1^{\circ} \mathrm{C}$ ). In the first step amounts of $2 \mathrm{ml}$ of each sol were mixed with $0.1 \mathrm{ml}$ of a $0.01 \mathrm{M}$ slurry of $4,4^{\prime}$-bis(2-benzoxazolyl)-stilbene (BOS) in ethanol. Fluorescence spectra were recorded in standard quartz-cuvettes with a Jobin Yvon Horiba FluoroMax-3 fluorescence spectrometer $\left(\lambda_{\text {ex }}=360 \mathrm{~nm}, \lambda_{\text {em }}=370-500 \mathrm{~nm}, \Delta \lambda=1 \mathrm{~nm}\right)$.

Automation of the above described experiment: doping of sols was carried out with a Tecan Genesis RSP 150 automated liquid handling system. $500 \mu \mathrm{l}$ portions of each sol were filled into $10 \mathrm{ml}$ glass test tubes and $25 \mu \mathrm{l}$ of a slurry of $0.01 \mathrm{M}$ BOS in ethanol (30 min ultrasonic treatment was applied to the BOS slurry before mixing with sol samples) were dispensed to each sol. The sol-BOS mixtures were stirred for $10 \mathrm{~min}$ and filled into a Multititerplate (MTP) with 96 wells. The spectroscopic high-throughput measurement was performed with a Tecan Spectrafluor microplate reader $\left(\lambda_{\mathrm{ex}}=360 \mathrm{~nm}, \lambda_{\mathrm{em}}=400 \mathrm{~nm}, 30\right.$ flashes per well, top reading mode).

\section{Results and discussion}

Spectroscopic measurements with viscosity sensitive fluorescence probes require samples with a Newtonian fluid behavior because no shear rate $D$ is applied during measurement. Representative dynamic viscosity $\eta(D)$ and sheer $\operatorname{stress} \tau(D)$ curves for a test sol containing $33.3 \mathrm{wt} \%$ 2propanol are shown in Fig. 1 and indicate an almost Newtonian behavior. Corresponding curves of other samples used in this study show similar characteristics and therefore admit the application of a spectroscopic viscosity measurement.

Fluorescence spectra of BOS doped nanocomposite sols acquired at $\lambda_{\mathrm{ex}}=360 \mathrm{~nm}$ are presented in Fig. 2. A previous excitation scan $\left(\lambda_{\mathrm{em}}=440 \mathrm{~nm}\right)$ led to a maximum intensity at $\lambda_{\mathrm{ex}}=370 \mathrm{~nm}$ but the constrained available excitation wavelengths of the fluorescence microplate reader later used for the high-throughput measurement necessitated

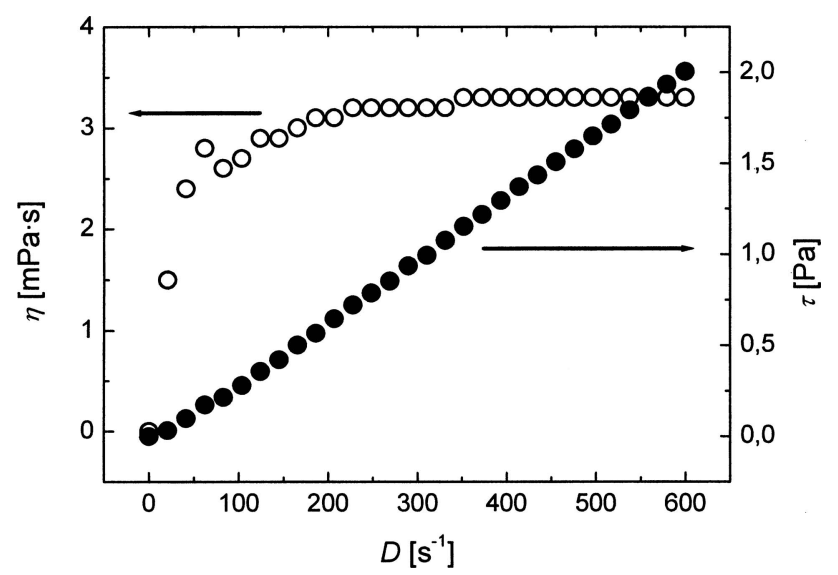

Fig. $1 \eta(D)$ and $\tau(D)$ curves from rotational viscometer measurement of a sol containing $33.3 \mathrm{wt}$ \% 2-propanol (sample 4)

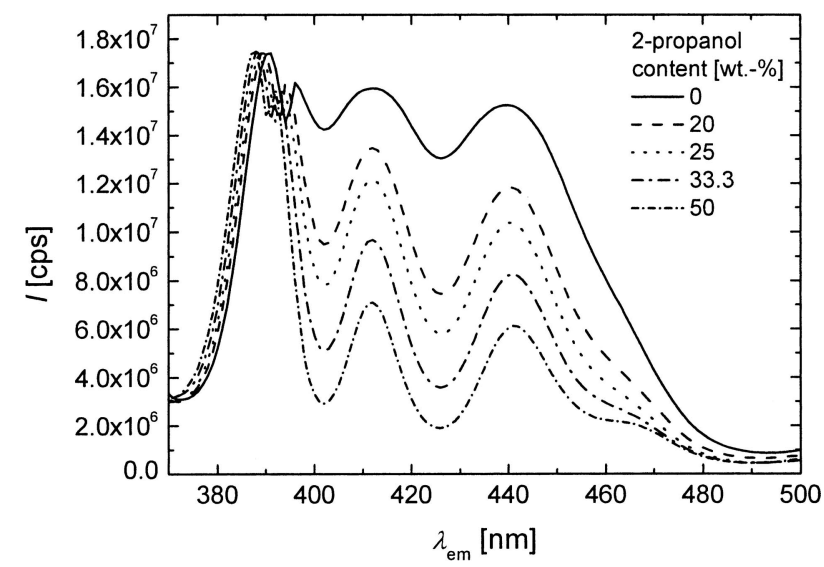

Fig. 2 Fluorescence spectra of 5 sols (see Table 1) doped with BOS slurry, viscosity sensitive bands at $\lambda_{\mathrm{em}}=412$ and $440 \mathrm{~nm}$, respectively. The spectra were recorded at $\lambda_{\text {ex }}=360 \mathrm{~nm}$, the BOS Concentration was in all samples $0.5 \mathrm{mmol} \cdot \mathrm{l}^{-1}$

a $\lambda_{\text {ex }}$ value of $360 \mathrm{~nm}$. According to Fig. 2 a satisfactory sensitivity was reached also at $\lambda_{\mathrm{ex}}=360 \mathrm{~nm}$. Maxima of viscosity sensitive bands of BOS are located at $\lambda_{\mathrm{em}}=412$ and $440 \mathrm{~nm}$, respectively. Furthermore, a non-viscosity sensitive peak with maximal intensity at $\lambda_{\mathrm{em}}=389 \pm 1.5 \mathrm{~nm}$ can be used as an internal standard to check for a uniform concentration of BOS in all samples. The intensity deviation at this position is $\leq 0.4 \%$ for all five samples and no additional intensity correction was necessary. All measurements were carried out at $T=20(1){ }^{\circ} \mathrm{C}$ to reduce shifts in intensity due to temperature dependency of fluorescence signals.

Viscosity sensitive intensity data at $\lambda_{\mathrm{em}}=412$ and $440 \mathrm{~nm}$ (Fig. 2) were considered for a calibration with viscosity data received from conventional rotational viscometer measurements. The relationship between fluorescence quantum yield $\Phi$ and viscosity $\eta$ is given by the Förster-Hoffmann equation and with assumption of a constant temperature given by Eq. (1) $[8]$

$\log \Phi=C+x \cdot \log _{\eta}$ 


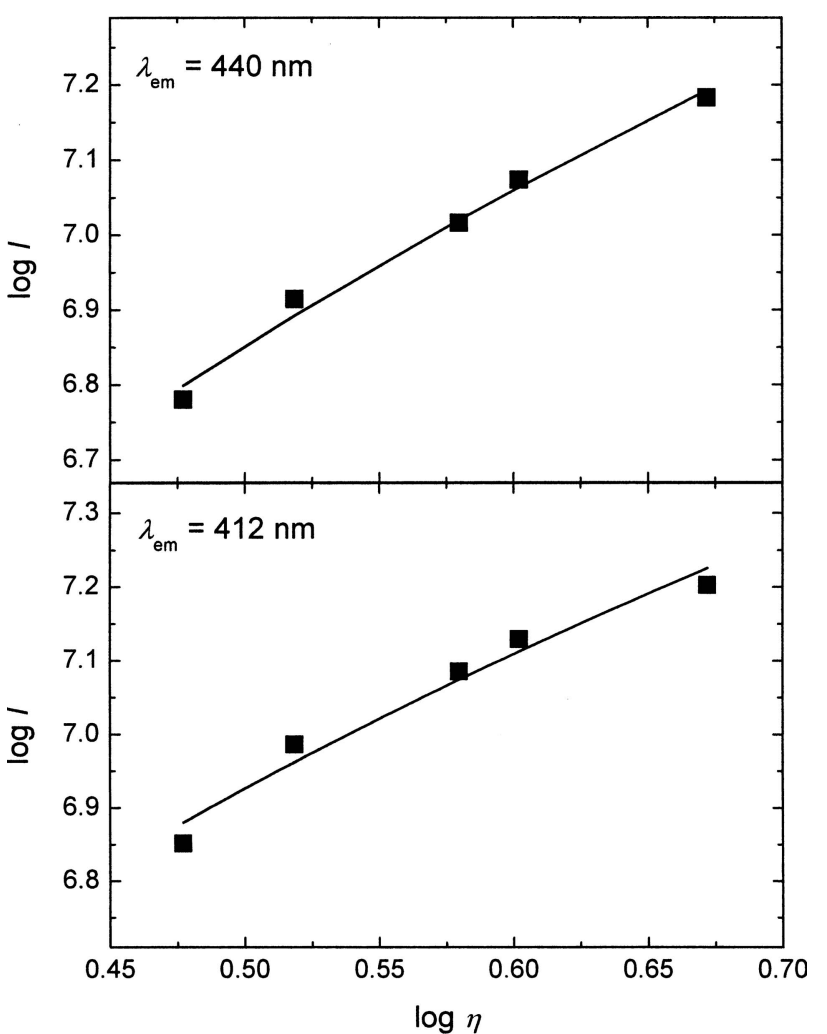

3 Calibration curves for achievement of constants $v$ and $\kappa$ (Eq. (2)) of intensity data at $\lambda_{\mathrm{em}}=440 \mathrm{~nm}$ (top) and $\lambda_{\mathrm{em}}=412 \mathrm{~nm}$ (bottom)

and assuming $\Phi \sim I$, constants $v$ and $\kappa$ were derived from a non-linear curve fitting from calibration Eq. (2) [4]

$\eta=(\kappa \cdot I)^{v}$

with constants $v$ and $\kappa$ related to constants $X$ and $C$ in Eq. (1) through

$v=1 / x ; \kappa=10^{-C}$

Data points of intensity over viscosity plotted in doublelogarithmic scale for calibration are presented in Fig. 3 and the non-linear fitting resulted in $v=0.14 \pm 0.01, \kappa=$ $7.65 \pm 0.07$, and correlation coefficient between mechanical and fluorescence-based viscosity values $R=0.96\left(\lambda_{\mathrm{em}}=\right.$ $412 \mathrm{~nm}$ ), and $v=0.16 \pm 0.01, \kappa=7.68 \pm 0.04, R=0.96$ $\left(\lambda_{\mathrm{em}}=440 \mathrm{~nm}\right)$, respectivel

High-throughput measurement

The transfer from sequential measurements using cuvettes ( $\geq 2 \mathrm{ml}$ sample) to parallel measurements using 96-well MTP's (150 $\mu$ l sample) is associated with a discrepancy in evolution of intensity data. Results of the high-throughput measurement are presented in Fig. 4. The multiplate fluorescence reader used in this study provides measurements

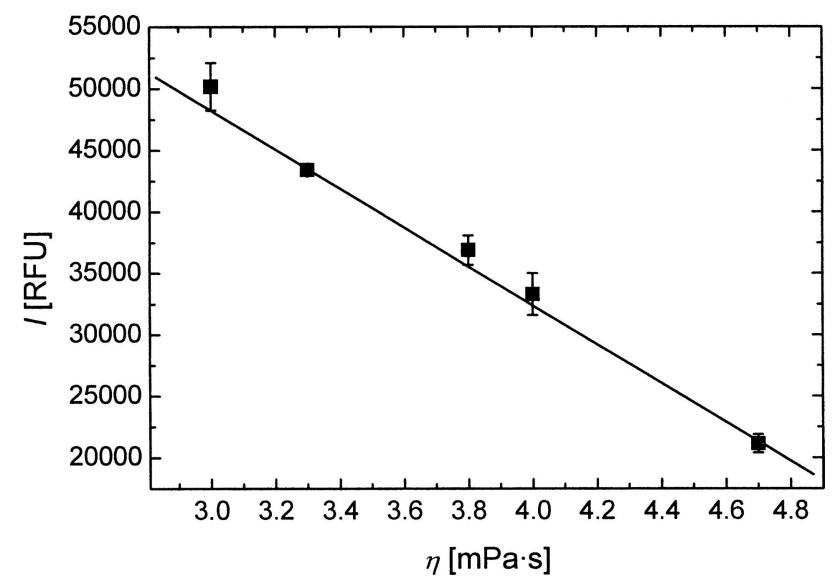

Fig. 4 Fluorescence data from microplate fluorescence reader plotted over viscosity data from mechanical viscosity measurements. The solid line represents a linear fit

at fixed $\lambda_{\mathrm{em}}$ using optical filters. The selected filter with $\lambda_{\mathrm{em}}=400 \mathrm{~nm}$ has a bandwidth of approx. $30 \mathrm{~nm}$ and therefore prevents a direct comparison of intensity data of viscosity sensitive fluorescence peaks acquired with fluorescence spectrometer (Fig. 2: $\lambda_{\mathrm{em}}=412$ and $440 \mathrm{~nm}$, respectively). Further, constrained acquisition of data with microplate reader does not allow a data fit according to the Förster-Hofmann Eq. (1) because of unknown contributions of non-viscosity sensitive fluorescence. Hence the dependency of $I(\eta)$ was expressed by a simple linear fit (Fig. 4) leading to the empirical correlation

$I(\eta)=95742-15834 \cdot \eta$

Error bars included in Fig. 4 indicate a reproducibility of single data points.

The above described high-throughput spectroscopic measurement of sol viscosities can be incorporated trouble-free into a workflow for an automated and parallel exploration of coating materials derived from nanocomposite sols.

\section{Conclusions}

The applicability of a spectroscopic viscosity measurement method for nanocomposite sols was demonstrated in this work. Intensity data of BOS doped sols showed a exponential correlation with viscosity values from mechanic measurements according to the Förster-Hoffmann Eq. (1). On the other hand, spectroscopic data recorded with a multiplate fluorescence reader for high-throughput purposes could not be interpreted with help of the Förster-Hoffmann Eq. (1) because of the contribution of non-viscosity sensitive fluorescence included in intensity data. Nevertheless, a simple linear correlation of fluorescence intensities and viscosity data from mechanical measurement could be established. 
A reasonable application of the high-throughput method for estimation of sol viscosities is filling a gap between sols prepared by conventional methods or an automated liquid handling system equipped with chambers for parallel reactions which provides reactands dissolved or in higher dilution for proper proportioning.

Acknowledgments This work was supported by BMBF (2A67/03 N 9040) and the State of Saarland.

\section{References}

1. C.W. Macosko, Rheology: Principles, Measurements, and Applications (VCH Weinheim, 1994), p. 181
2. L. Matsiev, J. Bennett, and E. McFarland, WO 9918431, 1999.

3. R.A. Potyrailo, D.R. Olson, G. Medford, and M.J. Brennan, Anal. Chem. 74, 5676 (2002).

4. M.A. Haidekker, A.G. Tsai, T. Brady, H.Y. Stevens, J.A. Frangos, E. Theodarkis, and M. Intaglietta, Am. J. Physiol. Heart Circ. Physiol. 282, 1609 (2002).

5. M.A. Haidekker, T. Ling, M. Anglo, H.Y. Stevens, J.A. Frangos, and E. A. Theodorakis, Chem. Biol. 8, 123 (2001).

6. K. Hakala, R. Vatanparast, S.Y. Li, C. Peinado, P. Bosch, F. Catalina, and H. Lemmetyinen, Macromolecules 33, 5954 (2000).

7. C. Lesniak, S. Brück, H. Schirra, and H. Schmidt, WO 0140394 , 2001.

8. T. Förster and G. Hoffmann, Z. Phys. Chem. 75, 63 (1971). 